$\xi$

\title{
Influence of Inclined Magnetic Field on the Peristaltic Flow of a Jeffrey Fluid in an Inclined Porous Channel
}

\author{
V. Jagadeesh ${ }^{1}$, S. Sreenadh ${ }^{1}$, P. Lakshminarayana ${ }^{2 *}$ \\ ${ }^{1}$ Department of Mathematics, Sri Venkateswara University, Tirupati, India \\ ${ }^{2}$ Department of Mathematics, School of Advanced Sciences, VIT, Vellore, India \\ *Corresponding author E-mail: lakshminarayana.p@vit.ac.in
}

\begin{abstract}
In this paper we have studied the effects of inclined magnetic field, porous medium and wall properties on the peristaltic transport of a Jeffry fluid in an inclined non-uniform channel. The basic governing equations are solved by using the infinite wave length and small Reynolds number assumptions. The analytical solutions have obtained for velocity and stream function. The variations in velocity for different values of important parameters have presented in graphs. The results are discussed for both uniform and non-uniform channels.
\end{abstract}

Keywords: Inclined magnetic field; Inclined porous channel; Jeffry fluid; Peristaltic flow, wall properties.

\section{Introduction}

Present days, the investigation of various types of non-Newtonian fluid flow problems has become an interesting research topic due to its numerous applications in science and technology. There is no unique model to describe the rheological behaviour of nonNewtonian fluids. Many researchers have studied several fluid models under various assumptions. Misra et al. [1] explains the effect of external magnetic fluid on a peristaltic transport of a biological fluid in an asymmetric channel through porous medium. Peristaltic pumping of a micropolar fluid in a tube was discussed by Srinivasacharya et al. [2]. Nandagopal et al. [3] investigated the influence of suction and injection on Couette flow of a Bingham fluid in a channel bounded by permeable beds. Micropolar fluid flow in an inclined channel bounded by permeable beds was studied by Nandagopal et al. [4]. Ellahi et al. [5] presented the series solutions to investigate the impact of slip conditions on heat transfer and magnetohydrodynamic flow of non-Newtonian nanofluid in a coaxial porous cylinder. Nandagopal et al. [6] described the flow of a non-Newtonian fluid sandwiched between two Newtonian fluids.

Peristalsis is a well-known mechanism of fluid transport by contraction and relaxation of a wave which is propagating on a channel walls. It has several physiological applications such as food transport by esophagus peristalsis, chyme movement due to intestine peristalsis, sperm transport in male reproductive tract etc. By applying this principle engineers have designed many industrial pumps to transport various types of fluids. In view of these applications, authors are presented different works on peristalsis. [7 $11]$.

Fluid flows through porous medium plays an important role in filtration process and recovery of the crude oil from the reservoir rocks and other applications. Similarly magnetohydrodynamic flows have many industrial and biomedical applications such as power generators, cancer treatment, drug delivery etc. Motivated by these facts investigators have tried to study the peristalsis by considering porous medium, external/induced magnetic field, wall properties etc. Akbar et al. [12] investigated the impact of slip and heat transfer on the Peristaltic flow of a third order fluid in an inclined asymmetric channel. The influence of slip boundary conditions and variable viscosity on the peristaltic transport of nonNewtonian fluid through an inclined porous channel was discussed by Ambreen Afsar Khan et al. [13]. The study of heat transfer and peristaltic slip flow of a Jeffrey fluid in a vertical asymmetric porous channel was examined by Lakshminarayana et al. [14]. Ramesh [15] discussed the heat and mass transfer along with the peristaltic flow of a couple stress fluid in the presence of inclined magnetic field in an inclined porous asymmetric channel. Heat transfer and Peristaltic transport of a Herschel-Bulkley fluid in an inclined channel with flexible walls was investigated by Sucharitha et al. [16]. Lakshminarayana et al. [17] studied the influence of Joule heating on the Peristaltic pumping of a Bingham fluid in an inclined porous channel.

In this paper we have investigated the peristaltic transport of a MHD Jeffrey fluid in an inclined porous channel having flexible walls. Long wave length and low Reynolds number approximations are used. The expressions for stream function and velocity are obtained. The effects of pertinent parameters on the fluid flow are discussed in detail.

\section{Mathematical Formulation}

A non-uniform inclined permeable channel of thickness $2 d$ is considered to study the peristaltic motion of a Jeffrey fluid. An inclined magnetic field is applied. The flow is unsteady and two dimensional which is formed by peristaltic wave with constant speed $c$. The geometry of the wall is considered as follows

$\mathrm{H}=\mathrm{d}+\overline{\mathrm{m}} \mathrm{x}+\mathrm{a} \operatorname{Sin} \frac{2 \pi}{\lambda}(\overline{\mathrm{x}}-\mathrm{ct})$

where $d, a, \lambda, \bar{m}$ and $t$ are the mean width, amplitude, wave length, non-uniformity of the channel and time respectively. 
The mass and momentum conservation equations are :

$\frac{\partial \mathrm{u}}{\partial \mathrm{x}}+\frac{\partial \mathrm{v}}{\partial \mathrm{y}}=0$

$$
\rho\left[\frac{\partial}{\partial \overline{\mathrm{t}}}+\overline{\mathrm{u}} \frac{\partial}{\partial \overline{\mathrm{x}}}+\overline{\mathrm{v}} \frac{\partial}{\partial \overline{\mathrm{y}}}\right] \overline{\mathrm{u}}=-\frac{\partial \overline{\mathrm{p}}}{\partial \overline{\mathrm{x}}}+\frac{\mu}{1+\lambda_{1}} \nabla^{2} \overline{\mathrm{u}}-\frac{\mu}{\mathrm{k}} \overline{\mathrm{u}}
$$$$
-\sigma \mathrm{B}_{0}{ }^{2} \cos \Theta(\overline{\mathrm{u}} \cos \Theta-\overline{\mathrm{v}} \sin \Theta)+\rho \mathrm{g} \sin \alpha,
$$

$\rho\left[\frac{\partial}{\partial \overline{\mathrm{t}}}+\overline{\mathrm{u}} \frac{\partial}{\partial \overline{\mathrm{x}}}+\overline{\mathrm{v}} \frac{\partial}{\partial \overline{\mathrm{y}}}\right] \overline{\mathrm{v}}=-\frac{\partial \overline{\mathrm{p}}}{\partial \overline{\mathrm{y}}}+\frac{\mu}{1+\lambda_{\mathrm{I}}} \nabla^{2} \overline{\mathrm{v}}-\frac{\mu}{\mathrm{k}} \overline{\mathrm{v}}$

$$
+\sigma \mathrm{B}_{0}^{2} \sin \Theta(\overline{\mathrm{u}} \cos \Theta-\overline{\mathrm{v}} \sin \Theta)-\rho \mathrm{g} \cos \alpha \text {, }
$$

where $\overline{\mathrm{u}}, \overline{\mathrm{v}}, \rho, \mu, \mathrm{p}, \sigma, \mathrm{B}_{0}, \mathrm{k}, \alpha, \Theta$ and $\mathrm{g}$ are the velocities in axial and transverse directions, density, fluid viscosity, pressure, electrical conductivity, magnetic field, permeability, inclination angle of the channel, inclination angle of the magnetic field and acceleration due to gravity.

The boundary conditions for this problem are given by $[16,17,18]$ :

$$
\begin{aligned}
& \frac{\partial}{\partial \overline{\mathrm{x}}} \mathrm{L}^{*}(\mathrm{H})=\frac{\partial \overline{\mathrm{p}}}{\partial \overline{\mathrm{x}}}=\frac{\mu}{1+\lambda_{\mathrm{I}}} \nabla^{2} \overline{\mathrm{u}}-\rho\left[\frac{\partial}{\partial \overline{\mathrm{t}}}+\overline{\mathrm{u}} \frac{\partial}{\partial \overline{\mathrm{x}}}+\overline{\mathrm{v}} \frac{\partial}{\partial \overline{\mathrm{y}}}\right] \overline{\mathrm{u}} \text { at } \overline{\mathrm{y}}=\mathrm{H}, \\
& -\frac{\mu}{\mathrm{k}} \overline{\mathrm{u}}-\sigma \mathrm{B}_{0}{ }^{2} \cos \Theta(\overline{\mathrm{u}} \cos \Theta-\overline{\mathrm{v}} \sin \Theta)+\rho \mathrm{g} \sin \alpha, \\
& \overline{\mathrm{u}}=0 \text { at } \overline{\mathrm{y}}=\mathrm{H}, \\
& \frac{\partial \overline{\mathrm{u}}}{\partial \overline{\mathrm{y}}}=0 \text { at } \overline{\mathrm{y}}=0,
\end{aligned}
$$

The velocity components are expressed in terms of stream function $\psi$ such that

$\overline{\mathrm{u}}=\frac{\partial \bar{\psi}}{\partial \mathrm{y}}$ and $\overline{\mathrm{v}}=-\frac{\partial \bar{\psi}}{\partial \mathrm{x}}$,

The dimensionless quantities are presented as follows:

$$
\left.\begin{array}{l}
\mathrm{x}=\frac{\overline{\mathrm{x}}}{\lambda}, \mathrm{y}=\frac{\overline{\mathrm{y}}}{\mathrm{d}}, \psi=\frac{\bar{\psi}}{\mathrm{cd}}, \mathrm{p}=\frac{\mathrm{d}^{2} \overline{\mathrm{p}}}{\mu \mathrm{c} \lambda}, \mathrm{t}=\frac{\mathrm{ct}}{\lambda}, \mathrm{m}=\frac{\lambda \overline{\mathrm{m}}}{\mathrm{d}}, \delta=\frac{\mathrm{d}}{\lambda}, \\
\varepsilon=\frac{\mathrm{a}}{\mathrm{d}}, \mathrm{M}=\sqrt{\frac{\sigma_{0}}{\mu}} \mathrm{B}_{0}, \sigma=\frac{\mathrm{d}}{\sqrt{\mathrm{k}}} \mathrm{h}=\frac{\mathrm{H}}{\mathrm{d}}=1+\mathrm{mx}+\varepsilon \sin 2 \pi(\mathrm{x}-\mathrm{t}), \\
\operatorname{Re}=\frac{\rho \mathrm{cd}}{\mu}, \mathrm{E}_{1}=\frac{-\tau \mathrm{d}^{3}}{\lambda^{3} \mu \mathrm{c}}, \mathrm{E}_{2}=\frac{\mathrm{m}_{1} \mathrm{~cd}^{3}}{\lambda^{3} \mu}, \mathrm{E}_{3}=\frac{\mathrm{cd}^{3}}{\lambda^{2} \mu}, \eta=\frac{\rho \mathrm{gd}^{2}}{\mu \mathrm{c}}
\end{array}\right\}
$$

where $\operatorname{Re}$ is the Reynolds number, $m$ is the non-uniform parameter, $\mathrm{M}$ is the magnetic parameter, $\sigma$ is the permeability parameter, $\delta$ is the wave number, $\varepsilon$ is the amplitude ratio, $\mathrm{E}_{1}, \mathrm{E}_{2}$ and $\mathrm{E}_{3}$ are the non-dimensional wall parameters.

\section{Solution of the Problem}

Using the very small Reynolds number and long wave length assumptions the dimensionless basic equations are presented as follows:

$$
\frac{1}{1+\lambda_{1}} \frac{\partial^{3} \psi}{\partial \mathrm{y}^{3}}-\left(\mathrm{M}^{2} \cos ^{2} \Theta+\sigma^{2}\right) \frac{\partial \psi}{\partial \mathrm{y}}+\eta \sin \alpha=\frac{\partial \mathrm{p}}{\partial \mathrm{x}}
$$

$0=\frac{\partial p}{\partial y}$

The corresponding dimensionless boundary conditions are (we assumed the zero value of the stream function at $\mathrm{y}=0$ )

$$
\begin{aligned}
& \frac{1}{1+\lambda_{1}} \frac{\partial^{3} \psi}{\partial \mathrm{y}^{3}}-\left(\mathrm{M}^{2} \cos ^{2} \Theta+\sigma^{2}\right) \frac{\partial \psi}{\partial \mathrm{y}}+\eta \sin \alpha \\
& =\left[\mathrm{E}_{1} \frac{\partial^{3} \mathrm{~h}}{\partial \mathrm{x}^{3}}+\mathrm{E}_{2} \frac{\partial^{3} \mathrm{~h}}{\partial \mathrm{x} \partial \mathrm{t}^{2}}+\mathrm{E}_{3} \frac{\partial^{2} \mathrm{~h}}{\partial \mathrm{x} \partial \mathrm{t}}\right] \\
& \psi=0 ; \quad \frac{\partial^{2} \psi}{\partial \mathrm{y}^{2}}=0 \quad \text { at } \mathrm{y}=\mathrm{h}, \\
& \frac{\partial \psi}{\partial \mathrm{y}}=0 \quad \text { at } \mathrm{y}=\mathrm{h},
\end{aligned}
$$

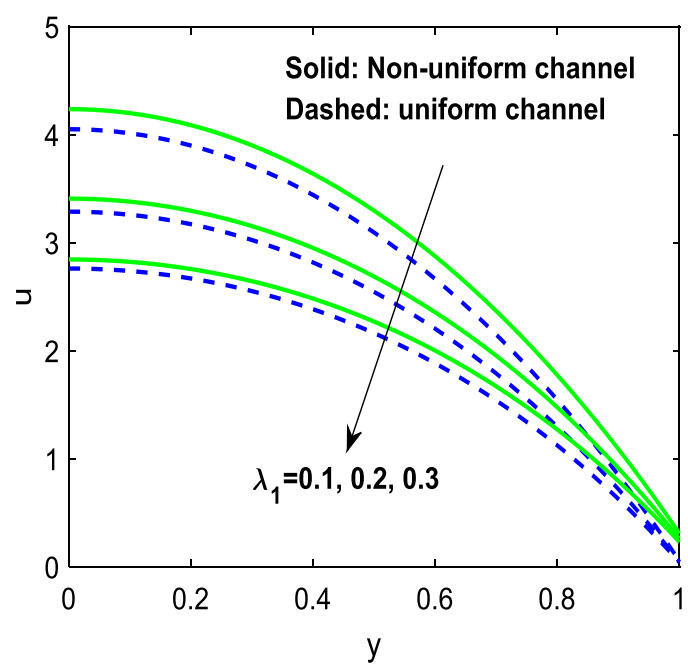

Fig.1: Velocity profiles for different values of $\lambda_{1}$

From equation (9) pressure is removed by cross differentiation and we obtain

$\frac{\partial^{4} \psi}{\partial \mathbf{y}^{4}}-\mathbf{M}_{1}^{2} \frac{\partial^{2} \psi}{\partial \mathbf{y}^{2}}=0$

where $\mathbf{M}_{1}^{2}=\left(1+\lambda_{1}\right)\left(\mathbf{M}^{2} \cos ^{2} \Theta+\sigma^{2}\right)$

We obtain the solution for stream function from equation (14) with the help of (11), (12) and (13)

$\psi=\frac{\mathrm{c}_{1}}{\mathrm{M}_{1}^{2}}\left[\frac{\sinh \mathrm{M}_{1} \mathrm{y}}{\mathrm{M}_{1} \cosh \mathrm{M}_{1} \mathrm{~h}}-\mathrm{y}\right]$

and the corresponding axial velocity is given by

$\mathrm{u}=\frac{\mathrm{c}_{1}}{\mathrm{M}_{1}^{2}}\left[\frac{\cosh \mathrm{M}_{1} \mathrm{y}}{\cosh \mathrm{M}_{1} \mathrm{~h}}-1\right]$

where 
$c_{1}=-8 \varepsilon \pi^{3}\left(E_{1}+E_{2}\right) \cos 2 \pi(x-t)+4 \varepsilon \pi^{2} E_{3} \sin 2 \pi(x-t)-\eta \sin \alpha$

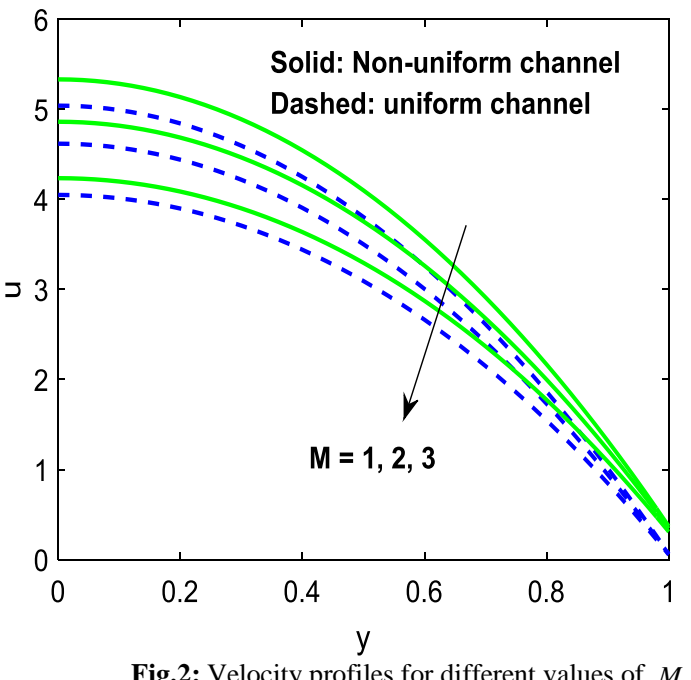

Fig.2: Velocity profiles for different values of $M$

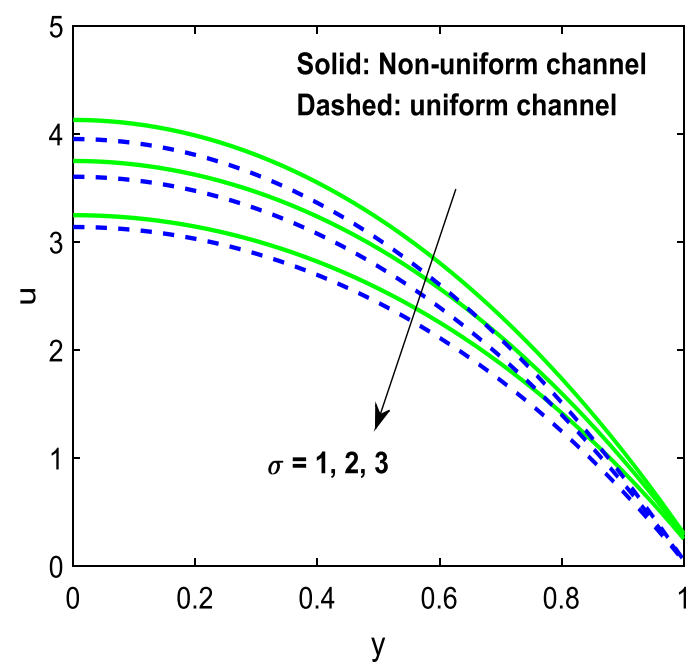

Fig.3: Velocity profiles for different values of $\sigma$

\section{Results and Discussion}

The effects of various parameters on the velocity field are explained graphically. We have plotted velocity profiles in figures Fig.1 - Fig.8 with the fixed values : $\mathrm{x}=0.15$, $\mathrm{t}=0.14, \mathrm{~m}=0,0.2, \varepsilon=0.2, \mathrm{M}=3, \sigma=1, \lambda_{1}=0.5, \mathrm{E}_{1}=0.2$, $\mathrm{E}_{2}=0.2, \mathrm{E}_{3}=0.1, \Theta=\frac{\pi}{6}, \alpha=\frac{\pi}{6}, \eta=1$. We have observed that the velocity of the Jeffrey fluid is more in the non-uniform channel when compared with the uniform channel. Fig. 1 is plotted to study the impact of Jeffrey parameter $\lambda_{1}$ on the velocity. Velocity profiles are parabolic. It is noticed that the velocity increases for large values of $\lambda_{1}$. Fig. 2 is drawn to study the effect of magnetic field on velocity. It is identified that the increase in magnetic parameter reduces the velocity of the fluid in both uniform and non-uniform channels. It shows the general phenomenon of Lorentz force. From fig. 3 it is noticed that the enhancing permeability parameter causes to low velocity of the fluid. This is the evidence of the physical property that the more permeability of the porous medium provides low resistance to the fluid flow.

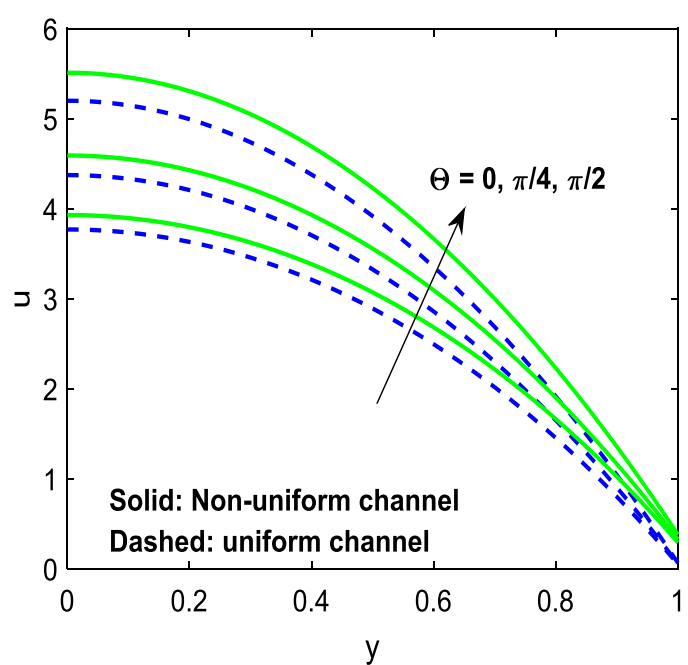

Fig.4: Velocity profiles for different values of $\Theta$

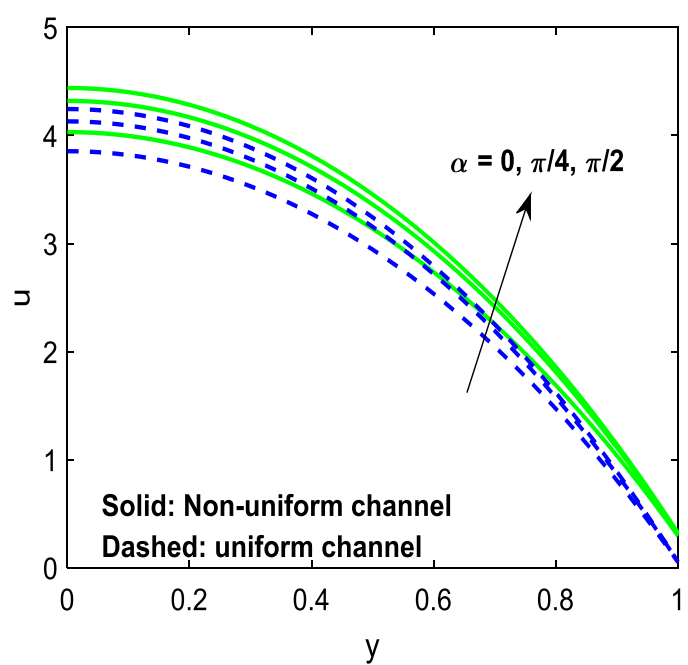

Fig.5: Velocity profiles for different values of $\alpha$

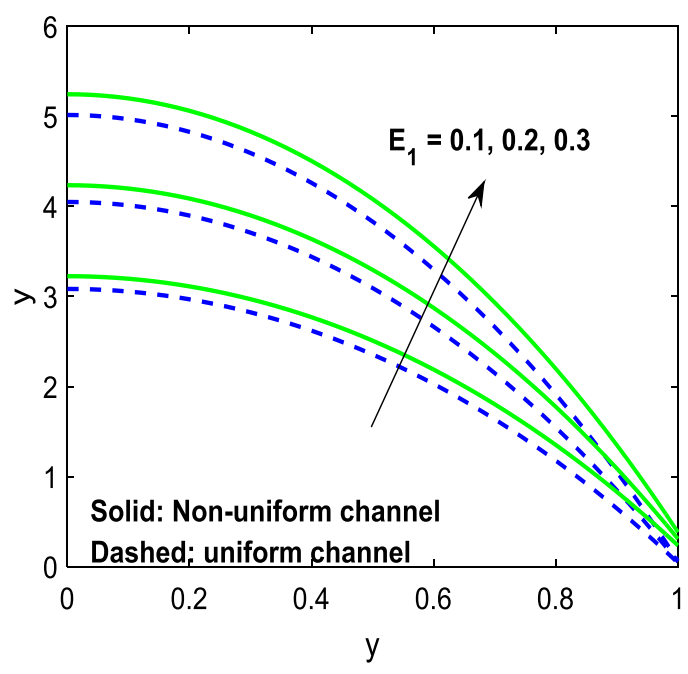

Fig.6: Velocity profiles for different values of $E_{1}$

Fig. 4 shows the influence of the inclination angle of the magnetic field. It is noticed that the velocity is the increasing function of $\Theta$. It shows the opposite behavior when compared with the magnetic parameter. Fig. 5 is made to observe the behavior of inclination angle of the channel. It is depicted that the rise $\alpha$ enhances the velocity of the fluid flow. The velocity profiles for different values of wall property parameters are drawn in figures in Fig. 6 - Fig. 8 . 
we have noticed that the velocity is an increasing function of wall tension parameter $\mathrm{E}_{1}$ and mass characterization parameter $\mathrm{E}_{2}$, while it is a decreasing function of damping force parameter $E_{3}$.

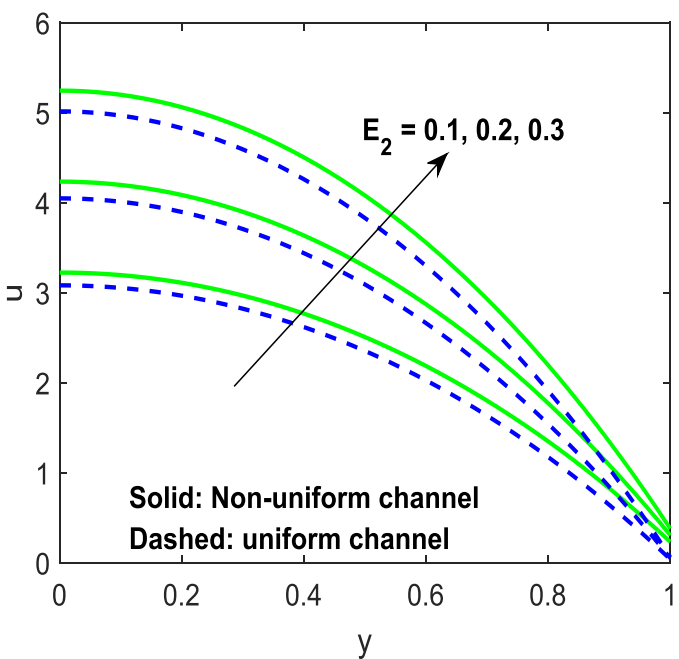

Fig.7: Velocity profiles for different values of $E_{2}$

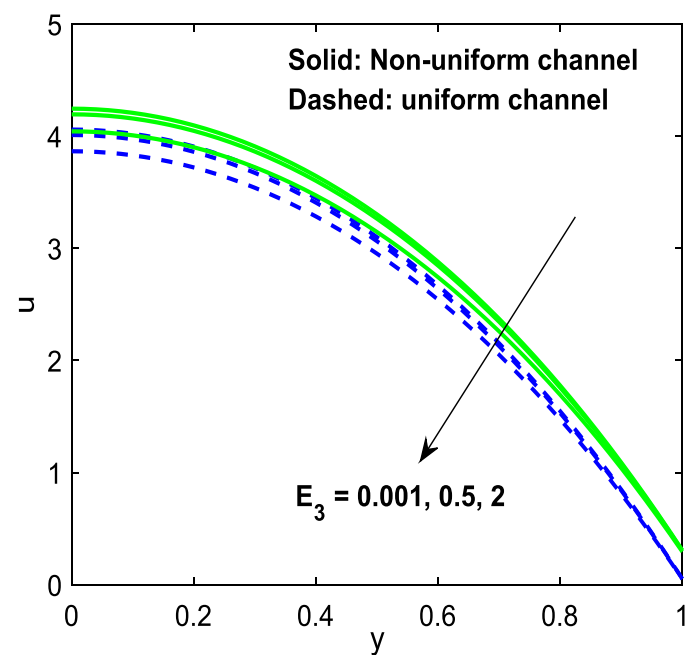

Fig.8: Velocity profiles for different values of $E_{3}$

\section{References}

[1] Misra JC, Maiti S \& Shit GC (2008) Peristaltic transport of a physiological fluid in an asymmetric porous channel in the presence of an external magnetic field. Journal of Mechanics in Medicine and Biology 8, (4) 507-525.

[2] Srinivasacharya D, Mishra M \& Ramachandra Rao A (2013) Peristaltic transport of a micropolar fluid in a tube. Acta. Mech. 16, 165-178.

[3] Nandagopal K, Sreenadh S, Lakshminarayana P \& Chakradhar K (2014) Couette flow of a Bingham fluid in a channel bounded by permeable beds with suction and injection. International Journal of Engineering Sciences \& Research Technology 3 (9), 300-305.

[4] Nandagopal K, Sreenadh S, Chakradhar K \& Lakshminarayana P (2015) Flow of a Micropolar fluid in an inclined channel bounded by permeable beds. International Journal of Applied Engineering Research 9 (20), 7691 - 7704.

[5] Ellahi R, Zeeshan A, Vafai K \& Rehman HU (2011) Series solutions for magnetohydrodynamic flow of non-Newtonian nanofluid and heat transfer in coaxial porous cylinder with slip conditions. $J$. Nanoeng. Nanosyst. 225, 123-132.

[6] Nandagopal K, Sreenadh S \& Lakshminarayana P (2016) flow of a non-Newtonian fluid sandwiched between two Newtonian fluids. International Journal of Mathematical Archive 7 (1), 137-146.

[7] Elshehawey EF, \& Mekhemier Kh S (1994) Couple-Stresses in peristaltic transport of fluids. J. Phys. D: Appl. Phys. 27, 1163.
[8] Srinivas S \& Kothandapani M (2009) The influence of heat and mass transfer on MHD peristaltic flow through a porous space with compliant walls. Appl. Math. Comput. 213, 197-208.

[9] Sankad G C \& Radhakrishnamacharya G (2009) Effect of magnetic field on peristaltic motion of a micropolar fluid with wall effects. $J$. Appl. Math. Fluid Mech. 1, 37-50.

[10] Satyanarayana KVV, Sreenadh S, Sucharitha G\& Lakshminarayana $P$ (2016) The effect of wall properties on the convective peristaltic transport of a conducting bingham fluid through porous medium. Indian Journal of Science and Technology 9 (42), 1-9.

[11] Vajravelu K, Sreenadh S, Dhananjaya S \& Lakshminarayana P (2016) Peristaltic flow and heat transfer of a conducting phan-thientanner fluid in an asymmetric channel - application to chyme movement in small intestine. Int. J. of Applied Mechanics and Engineering 21(3), 713-736.

[12] Akbar NS, Hayat T, Nadeem S \& Hendi A A (2011) Effects of slip and heat transfer on the Peristaltic flow of a third order fluid in an inclined asymmetric channel. Int. J. Heat Mass Transf. 54, 16541664.

[13] Ambreen Afsar Khan, Ellahi R \& Muhammad Usman (2013) The effects of variable viscosity on the peristaltic flow of nonNewtonian fluid through a porous medium in an inclined channel with slip boundary conditions. J. Porous Med. 16 (1), 59-67.

[14] Lakshminarayana P, Sreenadh S, Sucharitha G \& Nandagopal K (2015) Effect of slip and heat transfer on peristaltic transportof a Jeffrey fluid in a vertical asymmetric porous channel. Advances in Applied Science Research 6 (2), 107-118.

[15] Ramesh K (2016) Influence of heat and mass transfer on the peristaltic flow of a couple stress fluid through porous medium in the presence of inclined magnetic field in an inclined asymmetric channel. J. Mol. Liq. 219, 256-271.

[16] Sucharitha G, Vajravelu K, Sreenadh S \& Lakshminarayana P (2017) Peristaltic flow and heat transfer of a Herschel-Bulkley fluid in an inclined non-uniform channel with wall properties. IOP Conference Series: Materials Science and Engineering 263 (6), 062026.

[17] Lakshminarayana P, Vajravelu K, Sucharitha G \& Sreenadh S (2018) Peristaltic slip flow of a Bingham fluid in an inclined porous conduit with Joule heating. Applied Mathematics and Nonlinear Sciences 3 (1), 41-54.

[18] Sucharitha G, Rashidi M.M, Sreenadh S \& Lakshminarayana P (2018) Effects of magnetic field and slip on convective peristaltic

flow of a non-newtonian fluid in an inclined nonuniform porous channel with flexible walls. Journal of Porous Media 21 (10), 895-910. 